\title{
Circumflex Iliac Artery
}

National Cancer Institute

\section{Source}

National Cancer Institute. Circumflex Iliac Artery. NCI Thesaurus. Code C52863.

One of two (deep circumflex and superficial circumflex) iliac arteries that supply the lower abdomen, inguinal lymph nodes, sartorius, and tensor fasciae latae. 